\title{
Acute acquired toxoplasmosis: clinical-laboratorial aspects and ophthalmologic evaluation in a cohort of immunocompetent patients
}

\author{
ES Neves'1/, LN Bicudo1, AL Curi', E Carregal', WF Bueno', RG Ferreira', MR Amendoeira², \\ E Benchimol', O Fernandes ${ }^{3}$
}

${ }^{1}$ Instituto de Pesquisa Clínica Evandro Chagas ${ }^{2}$ Laboratório de Toxoplasmose ${ }^{3}$ Laboratório de Epidemiologia Molecular de Doenças Infecciosas, Instituto Oswaldo Cruz- Fiocruz, Av. Brasil 4365, 21045-900 Rio de Janeiro, RJ, Brasil

\begin{abstract}
Most cases of acute acquired toxoplasmosis (AAT) are oligosymptomatic and self-limited. Therefore, these infections rarely indicate treatment. Prospective studies of AAT patients are rare in the medical literature. The frequency of systemic manifestations has not been sufficiently studied. In order to search for risks factors for systemic and ocular involvement, 37 patients were submitted to a diagnostic investigative protocol. The most frequent findings were lymph node enlargement (94.6\%), asthenia (86.5\%), headache (70.3\%), fever (67.6\%) and weight loss (62.2\%). Hepatomegaly and/or splenomegaly were present in $21.6 \%$ of cases (8/37). Liver transaminases were elevated in 11 patients (29.7\%) and lactic dehydrogenase in 17 patients (45.9\%). Anaemia was found in four patients (10.8\%), leucopoenia in six patients (16.2\%), lymphocytosis in 14 patients (37.8\%) and thrombocytopenia in one patient (2.7\%). Fundoscopic examination revealed retinochoroiditis in four patients $(10.8 \%)$. No statistical association was found between any one morbidity and retinochoroiditis. Nevertheless, a significant association was found between the presence of more than eight morbidity features at evaluation and long-lasting disease. An ideal diagnostic protocol for AAT would include evidence of systemic involvement. Such a protocol could be used when planning treatment.
\end{abstract}

Key words: toxoplasmosis - retinochoroiditis - hepatomegaly - morbidity - Toxoplasma gondii

Toxoplasmosis is a zoonosis that is highly prevalent throughout the world. It is caused by Toxoplasma gondii, an intracellular coccidian parasite that infects several animal species (Montoya \& Liesenfeld 2004). Human infection occurs after ingestion of cysts from undercooked and contaminated meat. Alternatively, humans may ingest oocysts from contaminated water, soil and vegetables. After ingestion, gastric juices disrupt the outer wall of the cysts, releasing the infective forms. These forms reach the lymphatic system and blood circulation by dissemination through intestinal lumen cells. T. gondii invades all nucleated cells and tissues. Therefore, systemic involvement is frequent (Tenter et al. 2000). Human infection leads to an acute disease that is usually oligosymptomatic in immunocompetent individuals. The disease is considered to be self-limited and does not require treatment. Symptoms occur in $10-20 \%$ of cases (Montoya \& Liesenfeld 2004). The clinical manifestations include a mononucleosis-like syndrome, fever, lymph node enlargement, asthenia and headache.

Most previous studies have focused on congenital toxoplasmosis and immunocompromised patients. Prospective series of acute acquired toxoplasmosis (AAT) cases are rare in the literature (Moscatelli et al. 2006). Few studies have completed a systematic analysis of

Financial support: Fiocruz/IPEC, CNPq, Diagnósticos da América, Rio de Janeiro

+ Corresponding author: elizabeth.neves@ipec.fiocruz.br

Received 10 October 2008

Accepted 9 March 2009 the multi-organ involvement in AAT. The disease can attack the liver, spleen and retina. AAT can cause haematological changes (McCabe et al. 1987, Durlach et al. 2003). Anaemia, leucopoenia, lymphocytosis, thrombocytopenia, hepatitis and pneumonia may be secondary to AAT. However, these findings have been associated with rare, severe and disseminated disease (Bossi \& Bricaire 2004).

Retinochoroiditis was formerly associated with congenital disease. Recently, it has been recognised as a manifestation of post-natal infection (Couvreur \& Thulliez 1996, Burnett et al. 1998). Ocular lesions occur in up to $20 \%$ of infected patients. Lesions occur concomitant with an acute episode or after an unpredictable period. Lesions may occur as a unique episode with little inflammatory response. Repeated bouts of retinal lesions may lead to severe visual impairment (Montoya \& Remington 1996, Holland 1999).

This study describes a series of AAT patients who were submitted to a diagnostic investigative protocol (clinical evaluation, laboratory tests, abdominal ultrasound and fundoscopy). The protocol aimed to identify systemic involvement other than retinochoroiditis. A morbidity scale is proposed to provide better management for AAT patients.

\section{PATIENTS AND METHODS}

A longitudinal study including 37 patients (22 males and 15 females; $33 \pm 14$ years old) was performed. The cohort consisted of patients that attended Evandro Chagas Clinic Research Institute, Fiocruz, Rio de Janeiro. Patients were recruited from February 2006-August 2008. The final diagnosis of AAT was determined by (i) the presence of IgM anti-T. gondii $(>1.252 \mathrm{UI}$, posi- 
tive cut-off $>0.65$ ) and (ii) $\operatorname{IgG}$ antibodies with ascending titres (2 different samples at least two weeks apart) (ELISA Biokit, Toxo IgG and IgM, bioMérieux, Spain). To be included in the sampling, patients had to present with at least one of the following signs or symptoms of AAT: fever, lymphatic nodule enlargement, weight loss or retinochoroiditis. Avidity tests (IgG avidity ELISA, Diagnósticos da América, Brazil) were performed as definitive tests for the acute phase. Clinical-laboratory protocols included: (i) detailed anamnesis, (ii) physical examination, (iii) re-examination after two-four weeks, (iv) haemogram (Sysmex/Xt 1800i/Japan), (v) biochemical tests (liver function enzymes and serum lactic dehydrogenase (LDH) (Dimension/Dade, Behring/EUA), (vi) abdominal ultrasound (Phillips ENVISOR 521, 3$5 \mathrm{mhz}$ convex transducer, USA, same operator for all patients) and (vii) ophthalmologic examination (visual acuity, biomicroscopy and indirect ophthalmoscopy). Fundus photography was performed in the presence of retinochoroiditis. Those who presented with an abnormal ultrasound or ophthalmoscopic exam were re-examined with the same procedures during the follow-up visits. Ophthalmologic examinations were repeated every three months from six-30 months.

Based on the frequency of abnormal symptoms and tests (except serological tests), a morbidity scale was proposed using terciles. In this scale, patients with at least five points were classified as morbidity class I. Patients who presented with five-seven points were classified as morbidity class II. Patients with eight or more points were classified as morbidity class III.

Variables included in the scale were: (i) fever (axillary temperature $\geq 37.5^{\circ} \mathrm{C}$ ), (ii) lymph node enlargement, (iii) asthenia, (iv) headache, (v) weight loss of $>10 \%$, (vi) elevated liver transaminases (ALT $>65$ and AST $>45$ IU/l), (vii) elevated alkaline phosphatase (> $136 \mathrm{UI} / \mathrm{L}$ ), (viii) elevated LDH (> $190 \mathrm{UI} / \mathrm{L}$ ), (ix) anaemia (haemoglobin $<12 \mathrm{mg} / \mathrm{dL}$ in females and $<13 \mathrm{mg} /$ $\mathrm{dL}$ in males), (x) leucopoenia (leucocytes $<4 \times 10^{3} / \mu \mathrm{L}$ ), (xi) lymphocytosis (lymphocytes $>22 \%$ of total leucocytes), (xii) thrombocytopenia (platelets $<150 \times 10^{3} / \mu \mathrm{L}$ ) and (xiii) the presence of hepatomegaly and/or splenomegaly. The scale uses the reference values that are described in the instructions of the test kits. The scale was calculated based on patients (37/53) who attended the whole protocol.

Data were analysed using the Windows version of the SPSS.11.0 statistical software. The Spearman coefficient was used to verify the correlation between the morbidity scale, the presence of retinochoroiditis and long-lasting disease. Frequency tables and cross tabulation for different parameters were generated. Categorical data were analysed using Pearson's chi-square test to reveal any associations between the groups.

\section{RESULTS}

Population description and epidemiological data Among the 37 AAT patients referred to the Toxoplasmosis Outpatient Reference Clinic, 12 (32.4\%) came from public services, 10 (27\%) from private medical offices and $15(40.5 \%)$ from self-referral. The epidemiological profile of the analysed population is described in Table I. During the period of the study, 53 patients with AAT attended the Outpatient Unit of IPEC-Fiocruz. However, only 37 patients completed the follow-up protocol. Nevertheless, the entire population of patients presented with similar demographic characteristics.

\section{TABLE I}

Epidemiological profile of acute acquired toxoplasmosis patients

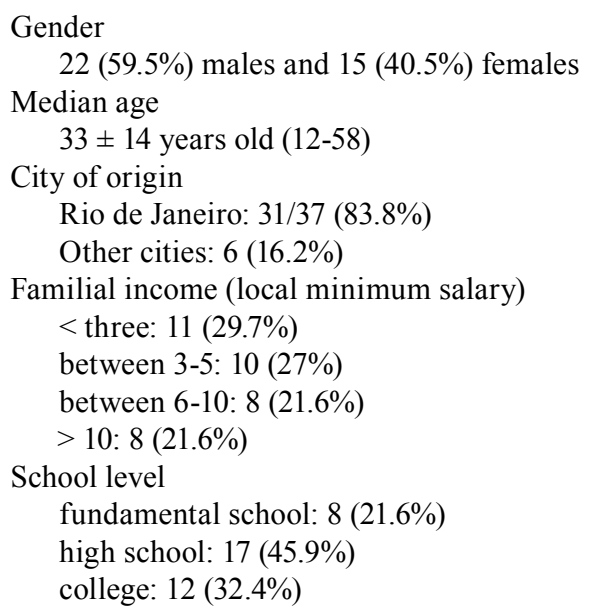

All patients provided at least one traditional epidemiologic risk factor for acquired toxoplasmosis (Tenter et al 2000). These factors included contact with cats $(45.9 \%)$, gardening $(29.7 \%)$, eating undercooked meat $(48.6 \%)$, eating raw salads $(86.5 \%)$, drinking tap water (51.4\%) and eating unwashed fruits (40.5\%).

Clinical and laboratorial features - At the first evaluation, no patients had coetaneous-mucosal lesions. Lymph node enlargement was the most commonly observed alteration $(35 / 37-94.6 \%)$. Hepatometry greater than $15 \mathrm{~cm}$ was observed in five individuals during their initial evaluation (5/37 - 13.5\%). A palpable spleen was found in one patient $(1 / 37-2.7 \%)$. Due to its sensitivity, abdominal ultrasound revealed liver enlargement in six patients $(6 / 37-16.2 \%)$ and splenomegaly in two patients $(2 / 37-5.4 \%)$. These ultrasonographic alterations were not observed after a two-three week interval. The main clinical and laboratory observations are displayed in Table II. No patients presented with abdominal lymph node enlargement.

Asthenia was observed in 32 patients ( $32 / 37-86.5 \%$ ). Within this group, $27(27 / 32-84.4 \%)$ patients reported symptoms lasting more than two weeks. Headaches were described by 26 AAT patients (26/37 - 70.3\%). Their intensity was reported using a visual analogue scale. Using this scale, significant headaches were found in 19 of the 26 aforementioned individuals $(19 / 26-73.1 \%)$. Fever occurred in $67.6 \%(25 / 37)$ of the patients. Fevers had lasted more than two weeks in 12 of the patients $(12 / 25$ - 48.0\%). Seven patients (7/37 - 18.9\%) presented with clinical findings (asthenia, headache and fever) for more 


\section{TABLE II}

Most common clinical and laboratory findings in the analyzed acute acquired toxoplasmosis population

\begin{tabular}{lc}
\hline Alterated data & $\begin{array}{c}\text { Frequency } \\
\mathrm{n}(\%)\end{array}$ \\
\hline Lymph nodes enlargement & $35(94.6)$ \\
Asthenia & $32(86.5)$ \\
Headache & $26(70.3)$ \\
Fever & $25(67.6)$ \\
Weigh loss & $23(62.2)$ \\
Liver transaminases elevated (ALT and/or AST) & $11(29.7)$ \\
Lactic dehydrogenase elevated & $17(45.9)$ \\
Anemia & $4(10.8)$ \\
Leucopenia & $6(16.2)$ \\
Lymphocytosis & $14(37.8)$ \\
Thombocytopenia & $1(2.7)$ \\
Hepatomegaly (US) & $6(16.2)$ \\
Splenomegaly (US) & $2(5.4)$ \\
\hline
\end{tabular}

TABLE III

Morbidity scale in acute acquired toxoplasmosis

\begin{tabular}{lc}
\hline Morbidity class & Frequency [n (\%)] \\
\hline I & $10(27)$ \\
II & $15(40.5)$ \\
III & $12(32.4)$ \\
\hline Total & $37(100)$ \\
\hline
\end{tabular}

than two weeks. Retinochoroiditis was observed in four (4/37 - 10.8\%) patients. No other major ophthalmological findings were discovered among the 37 examined individuals.

Using the results depicted in Table II, a morbidity scale (Table III) was constructed to identify the predictors of prolonged disease. This data was also used to detect associations between the severity of the disease and eye involvement.

Among the four individuals with retinochoroiditis, one was morbidity class I, two were morbidity class II and one was morbidity class III. No significant association was found between the intensity scale and the presence of retinochoroiditis $(\mathrm{p}=0.182)$. In addition, no association was found between each isolated variable or combinations of the variables and ocular involvement. Nevertheless, a correlation was found between the morbidity scale and long-lasting disease $(p<0.010)$. The morbidity scale correlated with the individual clinical parameters of prolonged disease, including asthenia $(\mathrm{p}<$ $0.001)$, fever $(\mathrm{p}<0.001)$ and headache $(\mathrm{p}<0.05)$.

\section{DISCUSSION}

Primary infection by $T$. gondii is considered to be a benign, oligosymptomatic and self-limiting disease (Montoya \& Liesenfeld 2004). Severe acute disseminated toxoplasmosis has been rarely described in immunocompetent patients (Bossi et al. 2002, Leal et al. 2007). Occasionally, normal patients develop long-last- ing symptoms. Until now, treatment has been based on subjective data. Prospective series of AAT patients are scarce in the literature. Most of the studies emphasise serological and histopathological characteristics (Durlach et al. 2003, Moscatelli et al. 2006). Clinical-laboratorial investigation of multiple-organ involvement of AAT has not been described in the literature. In Brazil, AAT cases are infrequent due to (i) the high seroprevalence for $T$. gondii infection and (ii) its common misdiagnose with non-specific febrile lymphadenopathy syndromes.

This series describes 37 ATT patients at a Brazilian public-infectious disease reference unit. The population involved is independent of any specific geographic region, school level or social class.

Altered haematological and biochemical blood parameters are rarely described in patients with AAT (McCabe et al. 1987, Moscatelli et al. 2006). In our cohort, lymphocytosis was the most common blood cell count alteration. Elevated serum LDH was a remarkable finding and may point to a sub-clinical pulmonary, myocardial or muscle involvement. The presence of liver enlargement and altered biochemical liver function tests indicates hepatic involvement, even in the absence of jaundice. Abnormalities in liver function have been noted by other authors (Tiwari et al. 1982, McCabe et al. 1987). It is worth noting the absence of abdominal lymph node enlargement. Other authors have emphasised the importance of cervical lymphadenopathy (Dorfman \& Remington 1973, McCabe et al. 1987). However, abdominal ultrasound screens have never been systematically carried out in AAT.

Toxoplasmosis is the most common cause of retinochoroiditis worldwide (Hovakimyan \& Cunningham 2002). Ocular toxoplasmosis was traditionally considered a manifestation of congenital and post-natal toxoplasmosis. However, in the last decade, researchers estimate that most of the ocular disease caused by $T$. gondi occurs due to post-natal infection (Holland 1999, Gilbert $\&$ Stanford 2000). Our study revealed a retinochoroiditis prevalence of $10.8 \%$ among the 37 AAT patients considered. In some Brazilian hyper-endemic areas, retinochoroiditis is described in up to $17 \%$ of AAT patients (Glasner et al. 1992). No national study has been performed in order to estimate ocular involvement in AAT. In other regions, the proportion of $T$. gondii-infected individuals with retinochoroiditis is approximately $2 \%$ (Holland 2003). In our cohort, no association was found between ocular disease and systemic disease. Considering these observations, routine fundoscopy is strongly recommended for all patients presenting with AAT. A longer follow-up and/or additional studies are required to better clarify this issue.

Toxoplasmosis is known to cause a mononuclear-like syndrome (Remington 1974, McCabe et al. 1987). The classic clinical features of this syndrome (asthenia, lymph node enlargement, fever and headache) were found in our series. In fact, asthenia was the most common symptom. Similar findings have been described by other authors (Durlach et al. 2003, Moscatelli et al. 2006).

In our series, a significant positive correlation was found between long-lasting disease and the morbidity scale. The morbidity scale was based on the clinical and 
laboratory parameters present at the initial patient evaluation. The correlation was significant when at least eight of the 13 proposed signs, symptoms and laboratory findings were positive. Better AAT patient management could be achieved if these parameters are considered. This scale may be useful when deciding about early treatment to shorten the acute clinical course of the disease. Statistical validation of this morbidity scale is still necessary.

None of the factors studied were associated with the development of ocular disease. Nevertheless, patients were followed for only 30 months. A longer follow-up study is being performed. In the future, patients will be re-examined at five-year intervals.

\section{ACKNOWLEDGEMENTS}

To David William Provance Junior, for helpful comments on the preparation of the manuscript.

\section{REFERENCES}

Bossi P, Bricaire F 2004. Severe acute disseminated toxoplasmosis. Lancet 364: 579.

Bossi P, Paris L, Caumes E, Katlama C, Danis M, Bricaire F 2002. Severe acute disseminated toxoplasmosis acquired by an immunocompetent patient in French Guyana. Scand J Infect Dis 34: 311-314.

Burnett AJ, Shortt SG, Isaac-Renton J, King A, Werker D, Bowie WR 1998. Multiple cases of acquired toxoplasmosis retinitis presenting in an outbreak. Ophthalmology 105: 1032-1037.

Couvreur J, Thulliez P 1996. Acquired toxoplasmosis of ocular or neurologic site: 49 cases. Presse Med 25: 438-442.

Dorfman RF, Remington JS 1973. Value of lymph node biopsy in the diagnosis of acute acquired toxoplasmosis. $N$ Engl J Med 289: 878-881.

Durlach RA, Kaufer F, Carral L, Hirt J 2003. Toxoplasmic lymphadenitis - clinical and serologic profile. Clin Microbiol Infect 9: $625-631$.
Gilbert RE, Stanford MR 2000. Is ocular toxoplasmosis caused by prenatal or postnatal infection? Br J Ophthalmol 84: 224-226.

Glasner PD, Silveira C, Kruszon-Moran D, Martins MC, Burnier Jr M, Silveira S, Camargo ME, Nussenblatt RB, Kaslow RA, Belfort Jr R 1992. An unusually high prevalence of ocular toxoplasmosis in southern Brazil. Am J Ophthalmol 114: 136-144.

Holland GN 1999. Reconsidering the pathogenesis of ocular toxoplasmosis. Am J Ophthalmol 128: 502-505.

Holland GN 2003. Ocular toxoplasmosis: a global reassessment. Part I: epidemiology and course of disease. Am J Ophthalmol 136: 973-988.

Hovakimyan A, Cunningham Jr ET 2002. Ocular toxoplasmosis. Ophthalmol Clin North Am 15: 327-332.

Leal FE, Cavazzana CL, Andrade Jr HF, Galisteo Jr AJ, Mendonça JS, Kallas EG 2007. Toxoplasma gondii pneumonia in immunocompetent subjects: case report and review. Clin Infect Dis 44: e62-e66.

McCabe RE, Brooks RG, Dorfman RF, Remington JS 1987. Clinical spectrum in 107 cases of toxoplasmic lymphadenopathy. Rev Infect Dis 9: 754-774.

Montoya JG, Liesenfeld O 2004. Toxoplasmosis. Lancet 363: 19651976.

Montoya JG, Remington JS 1996. Toxoplasmic chorioretinitis in the setting of acute acquired toxoplasmosis. Clin Infect Dis 23: 277-282.

Moscatelli G, Altcheh J, Biancardi M, Lapeña G, Ballering G, Freilij H 2006. Acutetoxoplasmosis: clinical and laboratory data in eleven patients. Ann Ped (Barc) 65: 551-555.

Remington JS 1974. Toxoplasmosis in the adult. Bull NY Acad Med 50: $211-227$

Tenter AM, Heckeroth AR, Weiss LM 2000. Toxoplasma gondii: from animals to humans. Int J Parasitol 30: 1217-1258.

Tiwari I, Rolland CF, Popple AW 1982. Cholestatic jaundice due to toxoplasma hepatitis. Postgrad Med J 58: 299-300. 